\title{
Direction of Arrival Estimation Accuracy Enhancement via Using Displacement Invariance Technique
}

\author{
Youssef Fayad, ${ }^{1}$ Caiyun Wang, ${ }^{2}$ Qunsheng Cao, ${ }^{1}$ and Alaa El-Din Sayed Hafez ${ }^{3}$ \\ ${ }^{1}$ College of Electronic and Information Engineering, Nanjing University of Aeronautics \& Astronautics, Nanjing 210016, China \\ ${ }^{2}$ College of Astronautics, Nanjing University of Aeronautics \& Astronautics, Nanjing 210016, China \\ ${ }^{3}$ Faculty of Engineering, Alexandria University, Alexandria 21615, Egypt
}

Correspondence should be addressed to Youssef Fayad; yousseffyad595@yahoo.com

Received 22 April 2015; Revised 2 August 2015; Accepted 5 August 2015

Academic Editor: Giuseppe Castaldi

Copyright (C) 2015 Youssef Fayad et al. This is an open access article distributed under the Creative Commons Attribution License, which permits unrestricted use, distribution, and reproduction in any medium, provided the original work is properly cited.

\begin{abstract}
A new algorithm for improving Direction of Arrival Estimation (DOAE) accuracy has been carried out. Two contributions are introduced. First, Doppler frequency shift that resulted from the target movement is estimated using the displacement invariance technique (DIT). Second, the effect of Doppler frequency is modeled and incorporated into ESPRIT algorithm in order to increase the estimation accuracy. It is worth mentioning that the subspace approach has been employed into ESPRIT and DIT methods to reduce the computational complexity and the model's nonlinearity effect. The DOAE accuracy has been verified by closedform Cramér-Rao bound (CRB). The simulation results of the proposed algorithm are better than those of the previous estimation techniques leading to the estimator performance enhancement.
\end{abstract}

\section{Introduction}

Direction of arrival Estimation (DOAE) for an antenna array is an important process because DOA is the creator of the tracking gate dimensions (the azimuth and the elevation). Accurate DOAE for the moving target leads to the reduction of the angle glint error which affects the accuracy of the tracking radars.

Doppler frequency shift is a significant phenomenon induced by the target movement. Lack of attention to the influence of Doppler shift on the ESPRIT method leads to DOAE process with poor accuracy.

The ESPRIT and its extracts have been widely studied in one-dimensional (1D) DOAE for uniform linear array (ULA) [1-4], nonuniform linear array (NULA) for spatial multiresolution [5-8], or temporal multiresolution [9-11] and also extended to two-dimensional (2D) DOAE [12-18]. All of these ESPRIT methods have been developed to upgrade the accuracy of DOAE with low calculation costs. However, these works did not pay attention to the effect of the Doppler frequency of the moving target on the DOAE accuracy. On the other hand, some other great efforts have been done to estimate Doppler frequency via using the fast Fourier transform (FFT), but it has a high order computation time for a large number of samples [19]. Another method applying ESPRIT technique to compute Doppler shift via employing the rotational factor resulted from time delay sampling [7, $8,20]$. However, these methods require intensive matrix computations or iterative optimization techniques.

This paper presents firstly a novel method to detect the target movement via estimating its Doppler frequency by comparing the displacement invariances of transmitter and receiver arrays, which enables the measurement of the sensor array displacement invariance fluctuation that resulted from wavelength change induced by the target movement. Secondly, for target moves with uniform acceleration, Doppler frequency correction has been embedded into the ESPRIT algorithm to refine the DOA estimated value. Subspace concept has been applied to reduce the model's nonlinearity effect and to realize parallel processing which leads to the enhancement of the estimation accuracy with low computational load $[5,6]$.

The paper is organized as follows. In Section 2, the twodimensions time multiresolution ESPRIT (2D T-ESPRIT) 


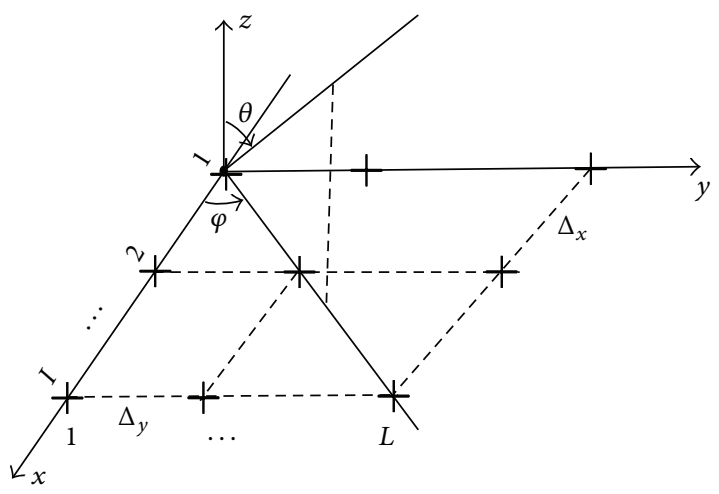

FIgURE 1: Planar antenna array.

DOAE technique has been introduced, the time multiresolution displacement invariance technique (T-DIT), and the Doppler effect of the moving target have been incorporated into the estimation algorithm. In Section 3, the simulation results are presented, and Section 4 is the conclusion.

\section{Proposed Algorithm}

2.1. The Measurement Model. In this model, the transmission medium is assumed to be isotropic and nondispersive, so that the radiation propagates in straight lines, and the sources are assumed to be as a far field away the array. Consequently, the radiation impinging on the array is a summation of the plane waves. The signals are assumed to be narrow-band processes, and they can be considered to be sample functions of a stationary stochastic process or deterministic functions of time [3]. Considering that there are $K$ narrow-band signals and the center frequency $f$ is assumed to have the same $\omega_{0}=$ $2 \pi f$, the $k$ th signal can be written as

$$
s_{k}(t)=E_{k} e^{j\left(\omega_{0} t+\Psi_{k}\right)}, \quad k=1,2, \ldots, K,
$$

where $s_{k}(t)$ is the signal of the $k$ th emitting source at time instant $t, \Psi_{k}$ is the carrier phase angles that are assumed to be random variables, each uniformly distributed on $[0,2 \pi]$ and all statistically independent of each other, and $E_{k}$ is the incident electric field that can be written as components form. As a general expression, we omit the subscript, and then

$$
\vec{E}=E_{\theta} \widehat{e}_{\theta}+E_{\varphi} \widehat{e}_{\varphi}
$$

where $E_{\varphi}$ and $E_{\theta}$ are the horizontal and the vertical components of the field, respectively.
Define $\gamma \in[0, \pi / 2]$ as the auxiliary polarization angle and $\eta \in[-\pi, \pi]$ as the polarization phase difference, and then,

$$
\begin{aligned}
& E_{\varphi}=|\vec{E}| \cos \gamma, \\
& E_{\theta}=|\vec{E}| \sin \gamma e^{j \eta} .
\end{aligned}
$$

The incident field can be also expressed in Cartesian coordinate system

$$
\begin{aligned}
\vec{E}= & E_{\theta} \widehat{e}_{\theta}+E_{\varphi} \widehat{e}_{\varphi} \\
= & \left(E_{\theta} \cos \theta \cos \varphi-E_{\varphi} \sin \varphi\right) \widehat{e}_{x} \\
& +\left(E_{\theta} \cos \theta \sin \varphi+E_{\varphi} \cos \varphi\right) \widehat{e}_{y}+\left(E_{\theta} \sin \theta\right) \widehat{e}_{z} .
\end{aligned}
$$

Figure 1 shows that a planar antenna array has elements indexed as $L, I$ along $y$ and $x$ directions, respectively. For any pair $(i, l)$, its coordinate is $(x, y)=\left((i-1) \Delta_{x},(l-1) \Delta_{y}\right)$, where $i=1, \ldots, I, l=1, \ldots, L$, and $\Delta_{x}$ and $\Delta_{y}$ are reference displacements between neighbor elements along $x$ - and $y$ axes. The array elements are oriented in xoy plane, and the space phase factors along $x$ and $y$ directions are expressed as

$$
\begin{aligned}
& p_{i}\left(\theta_{k}, \varphi_{k}\right) \equiv p_{i}^{k}=e^{j\left(2 \pi(i-1) \Delta_{x} / \lambda\right) \sin \theta_{k} \cos \varphi_{k}} \\
& q_{l}\left(\theta_{k}, \varphi_{k}\right) \equiv q_{l}^{k}=e^{j\left(2 \pi(l-1) \Delta_{y} / \lambda\right) \sin \theta_{k} \sin \varphi_{k}},
\end{aligned}
$$

where $\left(\theta_{k}, \varphi_{k}\right)$ denote the $k$ th source elevation angle and azimuth angle, respectively, and $\lambda$ is the wavelength of the $k$ th signal. The measurement vector can be expressed as

$$
\begin{aligned}
& z_{i, l}(t)=\sum_{k=1}^{K} u_{k} s_{k}(t) p_{i}\left(\theta_{k}, \varphi_{k}\right) q_{l}\left(\theta_{k}, \varphi_{k}\right)+w_{i, l}(t) \\
& {[Z(t)]} \\
& \quad=\left[\begin{array}{llllllll}
z_{1,1}(t) & \cdots & z_{1, L}(t) & \cdots & z_{I, 1}(t) & \cdots & z_{I, L}(t)
\end{array}\right]^{T},
\end{aligned}
$$

where

$$
\begin{aligned}
& {[W(t)]} \\
& \quad=\left[\begin{array}{llllllll}
w_{1,1}(t) & \cdots & w_{1, L}(t) & \cdots & w_{I, 1}(t) & \cdots & w_{I, L}(t)
\end{array}\right]^{T}
\end{aligned}
$$

stands for the additive white Gaussian noise (AWGN).

From (3) and (4), we got

$$
u_{k}=\left(\begin{array}{c}
\sin \gamma_{k} \cos \theta_{k} \cos \varphi_{k} e^{j \eta_{k}}-\cos \gamma_{k} \sin \varphi_{k} \\
\sin \gamma_{k} \cos \theta_{k} \sin \varphi_{k} e^{j \eta_{k}}+\cos \gamma_{k} \cos \varphi_{k}
\end{array}\right) .
$$

For receiving the array, the whole receiving factors in subspaces matrix are included in $\left[a\left(\theta_{k}, \varphi_{k}\right)\right]$; that is,

$$
a\left(\theta_{k}, \varphi_{k}\right) \stackrel{\text { def }}{=} p\left(\theta_{k}, \varphi_{k}\right) \otimes q\left(\theta_{k}, \varphi_{k}\right)
$$

where $\otimes$ denotes the Kronecker product. So

$$
A\left(\theta_{k}, \varphi_{k}\right)=\left[\begin{array}{llllllllllllllllllll}
u_{k}^{T} p_{1}^{k} q_{1}^{k} & u_{k}^{T} p_{1}^{k} q_{2}^{k} & \cdots & u_{k}^{T} p_{1}^{k} q_{L}^{k} & u_{k}^{T} p_{2}^{k} q_{1}^{k} & u_{k}^{T} p_{2}^{k} q_{2}^{k} & \cdots & u_{k}^{T} p_{2}^{k} q_{L}^{k} & \cdots & u_{k}^{T} p_{I}^{k} q_{1}^{k} & u_{k}^{T} p_{I}^{k} q_{2}^{k} & \cdots & u_{k}^{T} p_{I}^{k} q_{L}^{k}
\end{array}\right]^{T}
$$


The receiving model can be rewritten as,

$$
[Z(t)]=[A] S(t)+[W(t)]
$$

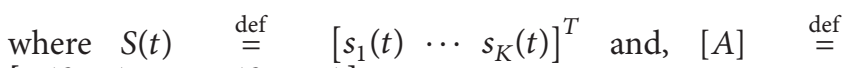
$\left[\begin{array}{lll}A\left(\theta_{1} \varphi_{1}\right) & \cdots & A\left(\theta_{K}, \varphi_{K}\right)\end{array}\right]$.

The subspace approach not only decreases the computational load as a result of shrinking the matrix dimensions, but also reduces the influence of nonlinearity when dealing with the signal inside each small time step as a linear part. Using the T-ESPRIT method, the whole data is divided into
$M$ snapshots at each time $t_{s}$ second with sampling frequency $f_{s}$. Then, it picks up enough data $r$ enclosed by each snapshot $m$ with time period $\tau=t_{s} / r$ as short as possible. So, from (11), each receiving signal measurement value through $m$ th subspace is given as

$$
\left[z^{m}(\tau)\right]=[A] s^{m}(\tau)+\left[w^{m}(\tau)\right] .
$$

The index $m$ runs as $m=1,2, \ldots, M$ snapshots. Therefore, the whole space-time steering data matrix can be expressed as

$$
Z \stackrel{\text { def }}{=}\left[\begin{array}{ccccccccccccc}
z_{1,1}^{1}(0) & \cdots & z_{1,1}^{1}\left(\tau_{1}\right) & \cdots & \cdots & z_{1,1}^{m}(0) & \cdots & z_{1,1}^{m}\left(\tau_{1}\right) & \cdots & \cdots & z_{1,1}^{M}(0) & \cdots & z_{1,1}^{M}\left(\tau_{1}\right) \\
\vdots & \ddots & \vdots & & & \vdots & \ddots & \vdots & & & \vdots & \ddots & \vdots \\
z_{I, L}^{1}(0) & \cdots & z_{I, L}^{1}\left(\tau_{1}\right) & \cdots & \cdots & z_{I, L}^{m}(0) & \cdots & z_{I, L}^{m}\left(\tau_{1}\right) & \cdots & \cdots & z_{I, L}^{M}(0) & \cdots & z_{I, L}^{M}\left(\tau_{1}\right)
\end{array}\right],
$$

where $\tau_{1}=(r-1) \times \tau$.

The dimension of $[Z]$ is $2(I \cdot L) \times M r$. For the $m$ th subspace, data matrix can be expressed as

$$
\left[z^{m}(\tau)\right]=\left[\begin{array}{ccc}
z_{1,1}^{m}(0) & \cdots & z_{1,1}^{m}\left(\tau_{1}\right) \\
\vdots & \ddots & \vdots \\
z_{I, L}^{m}(0) & \cdots & z_{I, L}^{m}\left(\tau_{1}\right)
\end{array}\right]
$$

For T-ESPRIT scheme, the ESPRIT algorithm is used in an appropriate picked data represented in (13) for each $(m)$ subspace, shown in (14), in parallel for the same sampling accuracy; thus, reducing the calculations load and consequently saving time is achieved.

2.2. Coarse DOAE Based on T-ESPRIT. The ESPRIT algorithm is based on a covariance formulation [2]; that is,

$$
\begin{aligned}
\widehat{R}_{z z} \stackrel{\text { def }}{=} E\left[Z(\tau) Z(\tau)^{H}\right] & =A \widehat{R}_{s s} A^{H}+\sigma^{2} \Sigma_{w}, \\
\widehat{R}_{s s} & =E\left[S(\tau) S^{H}(\tau)\right],
\end{aligned}
$$

where $\widehat{R}_{z z}$ is the correlation matrix of the array output signal matrix and $\widehat{R}_{z z}$ is the autocorrelation matrix of the signal. The subscript $H$ denotes the complex conjugate transpose.

The correlation matrix of $\widehat{R}_{z z}$ can be done for eigenvalue decomposition as follows:

$$
\widehat{R}_{z z} \stackrel{\text { def }}{=} \widehat{E}_{S} \Lambda \widehat{E}_{S}^{H}+\sigma^{2} \widehat{E}_{N} \Lambda \widehat{E}_{N}^{H}
$$

where the eigenvalues are ordered: $\lambda_{1}>\lambda_{2}>\cdots>\lambda_{K}>$ $\lambda_{K+1}>\lambda_{K+1}=\cdots=\lambda_{2(I \times L)}=\sigma^{2}$. The eigenvectors $\widehat{E}_{\mathrm{S}}=\left[\widehat{e}_{1}, \widehat{e}_{2}, \ldots, \widehat{e}_{K}\right]$ for larger $K$ eigenvalues span the signal subspace, and the rest $2(I \times L)-K$ smaller eigenvalues
$\widehat{E}_{N}=\left[\widehat{e}_{K+1}, \ldots, \widehat{e}_{2(I \times L)}\right]$ span the noise subspace which is orthogonal to the signal subspace. Therefore, there exists a unique nonsingular matrix $Q$, such that

$$
\widehat{E}_{S}=[A] Q \text {. }
$$

In (10), let $A_{P 1}$ and $A_{P 2}$ be the first and the last $2 L \times(I-$ 1) rows of $A$, respectively; they differ by the factor $\Delta p_{k}=$ $e^{j\left(2 \pi \Delta_{x} / \lambda\right) \sin \theta_{k} \cos \varphi_{k}}$ along the $x$ direction. So, $A_{P 2}=A_{P 1} \phi_{P}$, where $\phi_{P}$ is the diagonal matrix with diagonal elements $\Delta p_{k}$. Consequently, $\widehat{E}_{P 1}$ and $\widehat{E}_{P 2}$ will be the first and the last $2 L \times(I-1)$ submatrices formed from $\widehat{E}_{S}$. Then, the diagonal elements $p_{k}$ of $\phi_{P}$ are the eigenvalues of the unique matrix $\Psi_{P}=Q^{-1} \phi_{P} Q$ that satisfies

$$
\widehat{E}_{P 2}=\widehat{E}_{P 1} \Psi_{P}
$$

Similarly, two $2 I \times(L-1)$ submatrices $A_{q 1}$ and $A_{q 2}$ consist of the rows of $A$ numbered $2 L \times(i-1)+l$ and $2 L \times(i-$ 1) $+l+2$, respectively; they differ by the space factors $\Delta q_{k}=$ $e^{j\left(2 \pi \Delta_{y} / \lambda\right) \sin \theta_{k} \sin \varphi_{k}}$ along the $y$ direction; $l=1, \ldots, 2(L-1)$. Then, $A_{q 2}=A_{q 1} \phi_{q}$, where $\phi_{q}$ is the diagonal matrix with diagonal elements $\Delta q_{k}$. Consequently, $\widehat{E}_{S}$ forms the $2 I \times(L-1)$ two submatrices, $\widehat{E}_{q 1}$ and $\widehat{E}_{q 2}$. Then, the diagonal elements $\Delta q_{k}$ of $\phi_{q}$ are the eigenvalues of the unique matrix $\Psi_{q}=$ $Q^{-1} \phi_{q} Q$ that satisfies

$$
\widehat{E}_{q 2}=\widehat{E}_{q 1} \Psi_{q}
$$

Note that, for multiple signals processing, the ordering of the eigenvectors of $\Psi_{q}$ is generally permuted relative to the ordering of the eigenvectors of $\Psi_{P}$. So, it is essential to pair the eigenvalues of $\Psi_{q}$ and $\Psi_{P}$. Thus, for all possible permutations, the eigenvalue of $\Psi_{q}$ corresponding to $\Delta p_{k_{u}}$, the eigenvalue 
of $\Psi_{P}$, is the element from the $\Delta q_{k_{v}}$ set which realizes the following minimization:

$$
\left\{k_{1}^{0}, k_{2}^{0}, \ldots, k_{K}^{0}\right\}=\frac{\Delta p_{k_{u}}}{\Delta q_{k_{v}}}-\Delta q_{k_{t}},
$$

for $k_{u}, k_{v}, k_{t}=1,2, \ldots, K$

Therefore,

$$
\begin{aligned}
& \arg \left(\Delta p_{k}\right)=\frac{2 \pi \Delta_{x}}{\lambda} \sin \theta_{k} \cos \varphi_{k}, \\
& \arg \left(\Delta q_{k}\right)=\frac{2 \pi \Delta_{y}}{\lambda} \sin \theta_{k} \sin \varphi_{k} .
\end{aligned}
$$

And the coarse estimation of the arrival angles $\left(\theta_{k}, \varphi_{k}\right)$ can be calculated as

$$
\begin{aligned}
\theta_{k} & =\sin ^{-1}\left\{\frac { \lambda } { 2 \pi } \left[\left(\frac{\arg \left(\Delta p_{k}\right)}{\Delta_{x}}\right)^{2}\right.\right. \\
& \left.\left.+\left(\frac{\arg \left(\Delta q_{k}\right)}{\Delta_{y}}\right)^{2}\right]^{1 / 2}\right\}, \\
\varphi_{k} & =\tan ^{-1}\left[\frac{\Delta_{x}}{\Delta_{y}} \cdot \frac{\arg \left(\Delta q_{k}\right)}{\arg \left(\Delta p_{k}\right)}\right] .
\end{aligned}
$$

\subsection{Target Movement Detection}

2.3.1. T-DIT for Doppler Frequency Estimation. In T-DIT, we apply T-ESPRIT method also on a signal $\left(Z_{T}\right)$ generated from a transmitted signal sample and has its phase; then, we compare its displacement invariance with the displacement invariance of the received signal, which enables the Doppler frequency shift estimation via measuring the wavelength change.

Due to target movement, the arrival arguments of the displacement invariances for the colocated planar array at the receiver should be calculated as

$$
\begin{aligned}
& \underset{A}{\arg }\left(\Delta p_{k}\right)=\frac{2 \pi}{\grave{\lambda}_{x}} \Delta_{x} \sin \theta_{k} \cos \varphi_{k}, \\
& \underset{A}{\arg }\left(\Delta q_{k}\right)=\frac{2 \pi}{\grave{\lambda}_{y}} \Delta_{y} \sin \theta_{k} \sin \varphi_{k},
\end{aligned}
$$

where $\grave{\lambda}_{x}, \grave{\lambda}_{y}$ are the fluctuated wavelength components of the received wave into antenna plane and they differ from the transmitted wavelength because of the Doppler frequency $f_{d}$ caused by the target velocity $\vec{v}_{s}$ [21-23]. From Figure 2 , it is obvious that the wavelengths $\grave{\lambda}_{x}$ and $\grave{\lambda}_{y}$ have expressions caused by the velocity components $v_{x}$ and $v_{y}$ :

$$
\begin{aligned}
& \grave{\lambda}_{x}=\frac{\lambda\left(c+v_{x}\right)}{c}, \\
& \grave{\lambda}_{y}=\frac{\lambda\left(c+v_{y}\right)}{c} .
\end{aligned}
$$

TABLE 1: Relation between target direction and Doppler sign.

\begin{tabular}{lccc}
\hline Target direction & $\alpha$ & $\vec{v}_{s}$ & $f_{d}$ \\
\hline Away from antenna & $0 \leq \alpha<\pi / 2$ & + & - \\
Close to antenna & $\pi / 2<\alpha \leq \pi$ & - & + \\
\hline
\end{tabular}

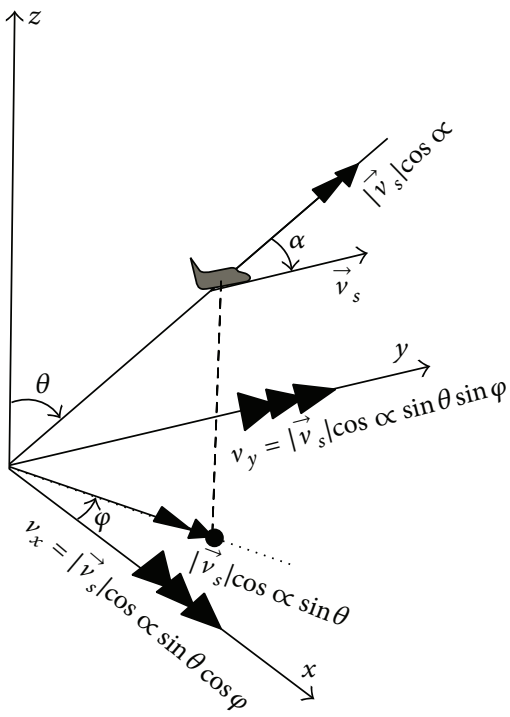

FIGURE 2: Target linear velocity components into antenna plane.

Substituting into (21),

$$
\begin{aligned}
& \underset{A}{\arg }\left(\Delta p_{k}\right)=\frac{2 \pi c}{\lambda\left(c+v_{x}\right)} \Delta_{x} \sin \theta_{k} \cos \varphi_{k}, \\
& \underset{A}{\arg }\left(\Delta q_{k}\right)=\frac{2 \pi c}{\lambda\left(c+v_{y}\right)} \Delta_{y} \sin \theta_{k} \sin \varphi_{k},
\end{aligned}
$$

where

$$
\begin{aligned}
& v_{x}=\left|\vec{v}_{s}\right| \cos \alpha \sin \theta_{k} \cos \varphi_{k}=\frac{c \cdot f_{d}}{2 f} \sin \theta_{k} \cos \varphi_{k}, \\
& v_{y}=\left|\vec{v}_{s}\right| \cos \alpha \sin \theta_{k} \sin \varphi_{k}=\frac{c \cdot f_{d}}{2 f} \sin \theta_{k} \sin \varphi_{k} .
\end{aligned}
$$

We define $c$ as a wave velocity in free space, and for a target moves with uniform acceleration $\alpha$ is the angle between the direction of wave propagation and the target velocity vector $\vec{v}_{s}$. Its value changes according to target maneuver. $f_{d}$ sign depends on $\alpha$ that indicates the target direction toward or away from the antenna position-target maneuver, as declared in Table 1.

For the transmitted signal, where there is no Doppler effect, the departure arguments are calculated as

$$
\begin{aligned}
& \underset{d}{\arg \left(\Delta p_{k}\right)}=\frac{2 \pi \Delta_{x}}{\lambda} \sin \theta_{k} \cos \varphi_{k}, \\
& \underset{d}{\arg \left(\Delta q_{k}\right)}=\frac{2 \pi \Delta_{y}}{\lambda} \sin \theta_{k} \sin \varphi_{k},
\end{aligned}
$$


TABLE 2: Target direction estimation cases.

\begin{tabular}{lccc}
\hline $\sin \left(\theta_{k}\right)$ & $v_{y}$ & $v_{y} \cdot \sin \left(\theta_{k}\right)$ & Target direction \\
\hline+ ve & + ve & $+v e$ & Away from antenna \\
+ ve & $-v e$ & $-v e$ & Close to antenna \\
- ve & $+v e$ & $-v e$ & Close to antenna \\
- ve & $-v e$ & $+v e$ & Away from antenna \\
\hline
\end{tabular}

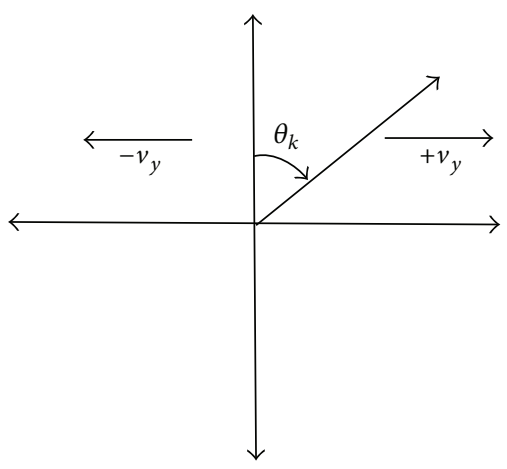

Figure 3: Target direction dependence on $v_{y}$ and $\theta_{k}$.

where departure and arrival angles are almost equal for the colocated case. From (26), (27), and (23)-(25), and due to time multiresolution as T-ESPRIT used in DOAE, the Doppler shift inside each time subspace for each target $\left(f_{d}^{k}\right)$ can be estimated as follows:

$$
\begin{aligned}
f_{d}^{k} & =\left(\frac{2 \cdot f}{\sin \theta_{k}}\right) \cdot\left[\left(\frac{\arg _{d}\left(\Delta p_{k}\right)}{\arg _{A}\left(\Delta p_{k}\right)}-1\right)^{2}\right. \\
& \left.+\left(\frac{\arg _{d}\left(\Delta q_{k}\right)}{\arg _{A}\left(\Delta q_{k}\right)}-1\right)^{2}\right]^{1 / 2} .
\end{aligned}
$$

And $\left(\theta_{k}\right)$ is the coarse estimated value of the elevation angle.

2.3.2. Target Direction Estimation. Obviously, it has been found from Figure 3 that target direction estimation depends on $v_{y}$ direction and $\theta_{k}$ value as indicated in Table 2.

It is clear that our main problem is to estimate $v_{y}$ sign to determine the target direction. By dividing (27) on (24), we have the following:

$$
v_{y}=c \cdot\left(\frac{\arg _{d}\left(\Delta q_{k}\right)}{\arg _{A}\left(\Delta q_{k}\right)}-1\right) .
$$

So,

$$
\begin{array}{ll}
\underset{A}{\arg }\left(\Delta q_{k}\right)<\underset{d}{\arg }\left(\Delta q_{k}\right), & \text { if } v_{y}=+\mathrm{ve}, \\
\underset{A}{\arg }\left(\Delta q_{k}\right)>\underset{d}{\arg }\left(\Delta q_{k}\right), & \text { if } v_{y}=-\mathrm{ve}, \\
\underset{A}{\arg }\left(\Delta q_{k}\right)=\underset{d}{\arg }\left(\Delta q_{k}\right), & \text { if } v_{y}=0,
\end{array}
$$

and $\grave{\lambda}_{x}=\grave{\lambda}_{y}=\lambda$ for stationary target.
2.4. Fine DOAE Based on Modified T-ESPRIT. The moving target echo signal is shifted by the Doppler effect. The more accurate T-ESPRIT algorithm should consider the effect of the Doppler frequency shift due to the target movement as mentioned in the previous section. Substitute by the Doppler frequency estimated value to refine angles estimated values as follows:

$$
\begin{aligned}
& \underset{A}{\arg }\left(\Delta p_{k}\right) \\
& =\frac{2 \pi c}{\lambda\left(c+\left(\left(c \cdot f_{d}^{k}\right) / 2 f\right) \sin \theta_{k} \cos \varphi_{k}\right)} \Delta_{x} \sin \theta_{k} \cos \varphi_{k}, \\
& \underset{A}{\arg }\left(\Delta q_{k}\right) \\
& =\frac{2 \pi c}{\lambda\left(c+\left(\left(c \cdot f_{d}^{k}\right) / 2 f\right) \sin \theta_{k} \sin \varphi_{k}\right)} \Delta_{y} \sin \theta_{k} \sin \varphi_{k}, \\
& \underset{A}{\arg }\left(\Delta p_{k}\right)+\underset{A}{\arg }\left(\Delta p_{k}\right) \cdot\left(\frac{f_{d}^{k}}{2 f}\right) \sin \theta_{k} \cos \varphi_{k} \\
& =\left(\frac{2 \pi \Delta_{x}}{\lambda}\right) \cdot \sin \theta_{k} \cos \varphi_{k} \\
& \underset{A}{\arg }\left(\Delta q_{k}\right)+\underset{A}{\arg }\left(\Delta q_{k}\right) \cdot\left(\frac{f_{d}^{k}}{2 f}\right) \sin \theta_{k} \sin \varphi_{k} \\
& =\left(\frac{2 \pi \Delta y}{\lambda}\right) \cdot \sin \theta_{k} \sin \varphi_{k} \text {. }
\end{aligned}
$$

So,

$$
\begin{aligned}
& \underset{A}{\arg }\left(\Delta p_{k}\right)=\sin \theta_{k} \cos \varphi_{k} \cdot\left[\left(\frac{2 \pi \Delta_{x}}{\lambda}\right)-\left(\underset{A}{\arg }\left(\Delta p_{k}\right)\right.\right. \\
& \left.\left.\cdot\left(\frac{f_{d}^{k}}{2 f}\right)\right)\right]
\end{aligned}
$$$$
\underset{A}{\arg }\left(\Delta q_{k}\right)=\sin \theta_{k} \sin \varphi_{k} \cdot\left[\left(\frac{2 \pi \Delta y}{\lambda}\right)-\left(\underset{A}{\arg }\left(\Delta q_{k}\right)\right.\right.
$$$$
\left.\left.\cdot\left(\frac{f_{d}^{k}}{2 f}\right)\right)\right]
$$$$
\theta_{k}=\sin ^{-1}\left\{\left[\left(\frac{\arg _{A}\left(\Delta p_{k}\right)}{\left(2 \pi \Delta_{x} / \lambda\right)-\left(\arg _{A}\left(\Delta p_{k}\right) \cdot\left(f_{d}^{k} / 2 f\right)\right)}\right)^{2}\right.\right.
$$$$
\left.\left.+\left(\frac{\arg _{A}\left(\Delta q_{k}\right)}{\left(2 \pi \Delta_{y} / \lambda\right)-\left(\arg _{A}\left(\Delta q_{k}\right) \cdot\left(f_{d}^{k} / 2 f\right)\right)}\right)^{2}\right]^{1 / 2}\right\}
$$

$$
\varphi_{k}=\tan ^{-1}\left[\frac{\arg _{A}\left(\Delta q_{k}\right)}{\arg _{A}\left(\Delta p_{k}\right)}\right.
$$

$$
\left.\cdot \frac{\left(2 \pi \Delta_{x} / \lambda\right)-\left(\arg _{A}\left(\Delta p_{k}\right) \cdot\left(f_{d}^{k} / 2 f\right)\right)}{\left(2 \pi \Delta_{y} / \lambda\right)-\left(\arg _{A}\left(\Delta q_{k}\right) \cdot\left(f_{d}^{k} / 2 f\right)\right)}\right] \text {. }
$$




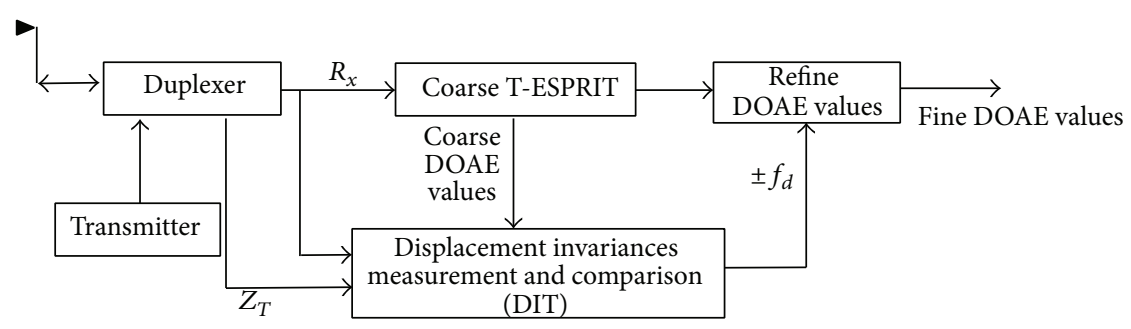

(a)

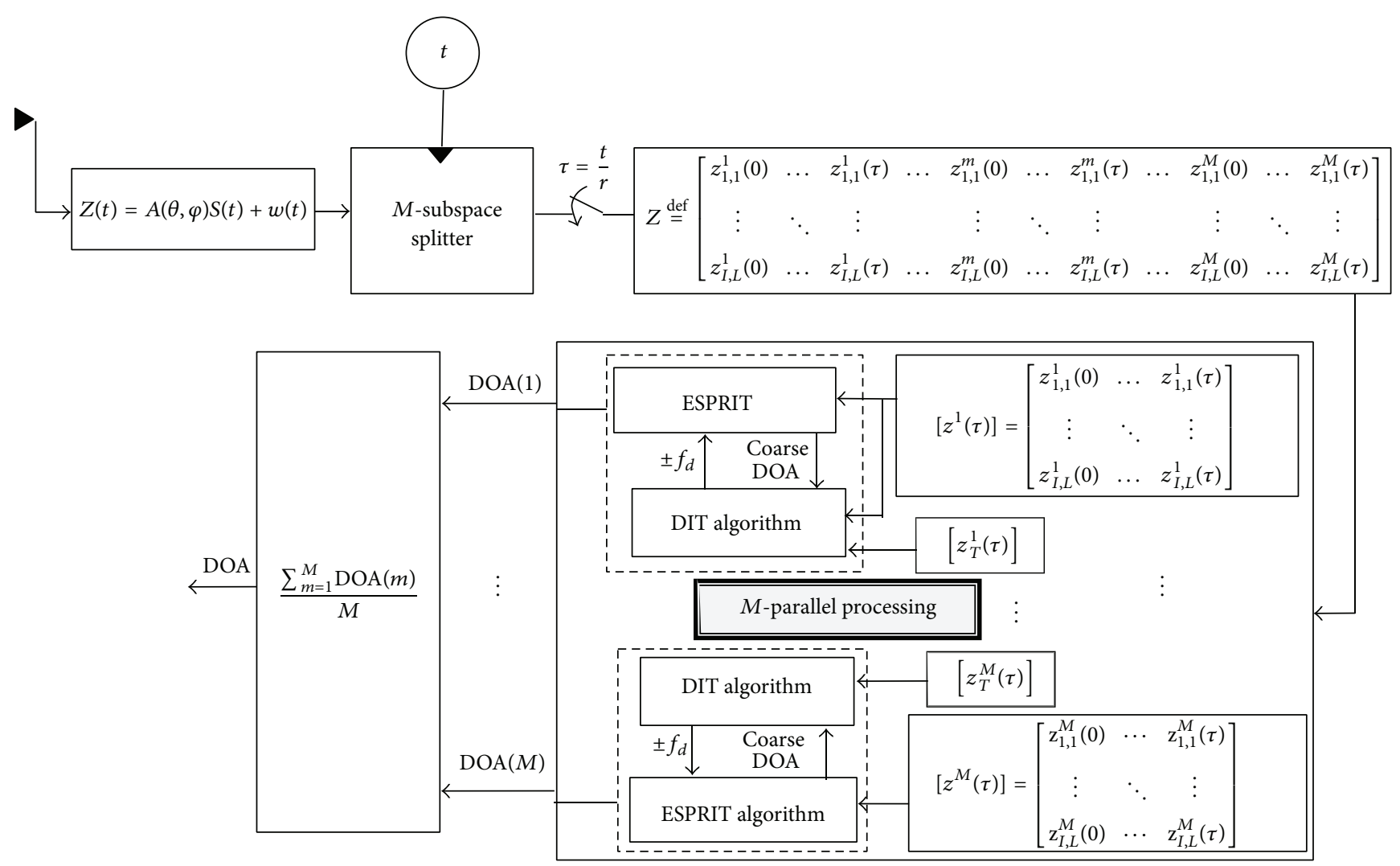

(b)

FIgURE 4: T-DIT and Doppler correction for T-ESPRIT algorithm (a) general scheme and (b) detailed scheme.

TABLE 3: Comparison of the required computation time and complexity.

\begin{tabular}{lcc}
\hline Comparison item & Algorithm & Proposed algorithm \\
\hline $\begin{array}{l}\text { Computational time (msec) } \\
\text { Computational complexity }\end{array}$ & Conventional ESPRIT & 10.2 \\
\hline
\end{tabular}

It is obviously found from (33) that if $f_{d}=0$, it will realize a stationary target indicated in (20). Figures 4(a) and 4(b) show the use of T-DIT to refine DOAE process.

\section{Simulation Results and Comparisons}

Considering the 2D-DOAE process with the additive white Gaussian noise (AWGN), the parameters are given; $f_{s}$ $=25 \mathrm{MHz}$. Assume total 25 temporal snapshots, pickup enclosed data $r=20$ times, $v_{s}=250 \mathrm{~m} / \mathrm{sec}$, and $f=3 \mathrm{GHz}$.
A received signal model is simulated using 200 independent Monte Carlo simulations. In order to validate the T-ESPRIT method, it has been used in the planar case with number of elements, such as $(I, L)=(8,8)$, with displacement values $\Delta_{x}=\Delta_{y}=\lambda / 2$, and with initial values of $\theta=45^{\circ}$ and $\varphi=60^{\circ}$. It is found that the computational load was reduced as a result of reducing the measurement matrix dimension to $(2 I L \times r)$ instead of $(2 I L \times M r)$ and employing the subspaces parallel processing concept. Table 3 represents the computational time and complexity of the proposed 


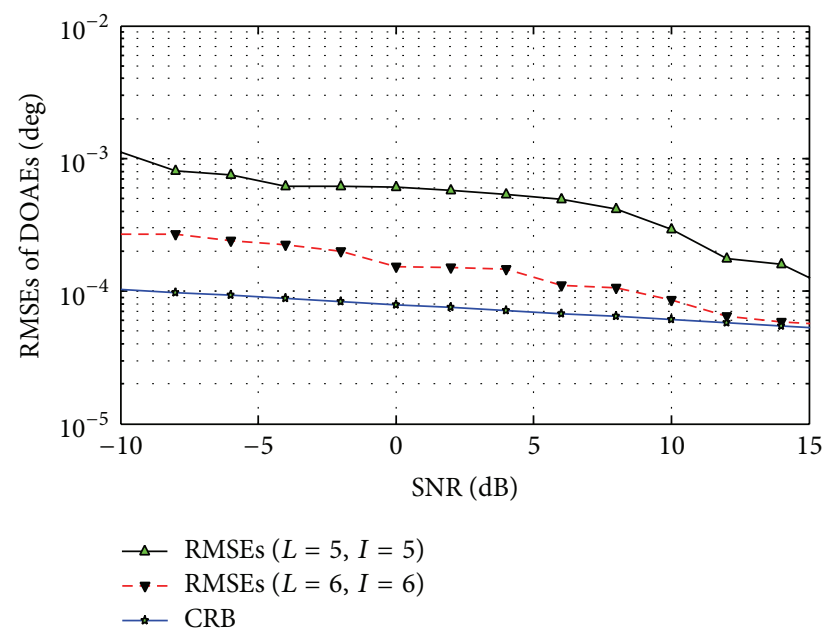

FIGURE 5: RMSEs versus SNR of T-ESPRIT with different number of elements for stationary target.

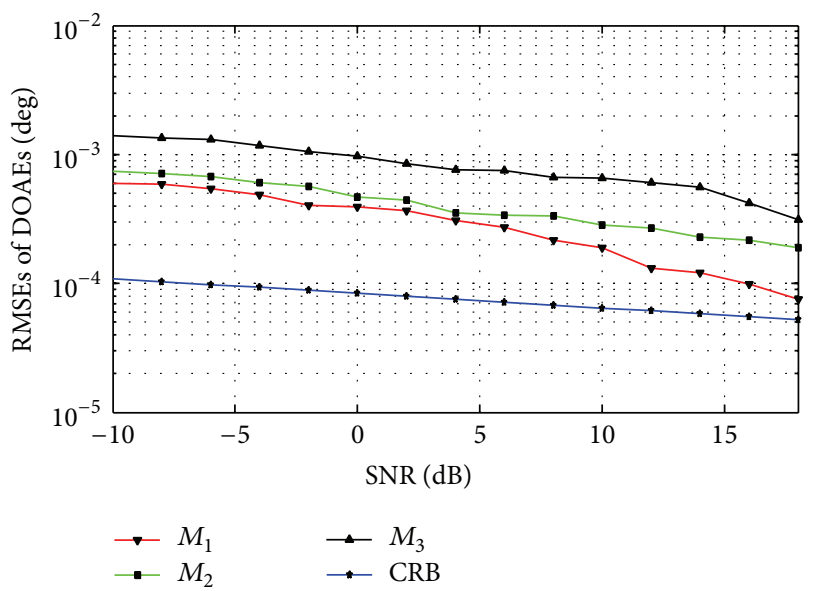

FIGURE 6: RMSEs versus SNR of T-ESPRIT for different maneuver cases.

T-ESPRIT method in terms of number of flip-flops. It is obvious that the computational load has been reduced as a result of a simultaneous processing for $M$ subspaces with each having $r$ snapshots instead of processing for one space that has a large number of snapshots $d,(d=M r)$ snapshots.

Figure 5 plotted the RMSEs for stationary target with different numbers of elements. The results in Figure 5 indicate that the errors are getting closer to the CRB due to the increase in the number of elements.

Figure 6 showed the errors of the T-ESPRIT method for different maneuver cases according to $\alpha$. For simplicity, we pointed to the three cases by $M_{1}, M_{2}$, and $M_{3}$ due to the different three values of $\alpha$, where $M_{1}$ is the maneuver at $\alpha=$ $0^{\circ}, M_{2}$ is the maneuver at $\alpha=95^{\circ}$, and $M_{3}$ is the maneuver at $\alpha=89^{\circ}$ (the highest maneuver because $\alpha$ is close to $90^{\circ}$ ). The displayed results showed that the lowest maneuver $\left(M_{1}\right)$ RMSEs curve is the closest to CRB $[24,25]$ on the contrary to the highest maneuver $\left(M_{3}\right)$ RMSEs curve which clarify the effect of the target movement on the estimation accuracy.
TABLE 4: Comparison of the required computation time.

\begin{tabular}{lc}
\hline Algorithms & Computation time $(\mathrm{Sec})$ \\
\hline Direct calculation & 80.3702 \\
FFT method & 1.5112 \\
ZFFT method & 0.1604 \\
PWZFFT method & 0.0835 \\
Proposed T-DIT method & 0.00926 \\
\hline
\end{tabular}

Results in Figures 5 and 6 indicate that the RMSEs of the stationary target DOAE are more close to the CRB even in low SNR, but, in case of moving targets as shown in Figure 6, the RMSEs of the maneuvering target tend to be closer to the CRB with higher SNR.

The T-DIT method has been used to estimate Doppler frequency via measuring the fluctuation of the sensor array displacement invariance while the method used in [20] employed the rotational factor that resulted from time delay. Figure 7 showed that the T-DIT algorithm results are better than those of [20] algorithm. This improvement was due to the decrease of the errors caused by the model nonlinearity effect. Additionally, in order to introduce the improvement in the computation time compared to estimate Doppler frequency via using the FFT method, Table 4 represents a comparison between the proposed method computation time and the different FFT methods mentioned in [26].

The results in Table 4 show that the computation time is reduced to $11.09 \%$ compared to the best computation time which has been realized via using PWZFFT method [26]. This improvement has been realized because of using the temporal subspaces concept which enables parallel processing.

Note that the Doppler correction for 2D-DOAE may increase the total computational time which is consumed to obtain the 2D-DOAE. The computational time of the 2D-DOAE with Doppler correction implementation is $(10.2 \mathrm{msec})$ as indicated in Table 3 . However, the computational time is still $57.63 \%$ of the conventional ESPRIT computational time.

Figure 8 showed the T-ESPRIT errors with $f_{d}$ embedding at $\alpha=0^{\circ}\left(f_{d}=5000 \mathrm{~Hz}\right)$. The accuracy improvement of the 2D-DOAE using proposed algorithm (T-ESPRIT with $f_{d}$ embedding) has been verified by comparing the resulted RMSEs with the RMSEs of the 2D Beam ESPRIT, the quaternion ESPRIT algorithms, and the ESPRIT-Like used in [14], [15], and [16], respectively; Figure 8 shows this comparison results.

Comparison results displayed in Figure 8 show that the proposed algorithm has a better performance, especially at a low SNR. This upgrade has been realized firstly due to the increase of DOAE accuracy when using the T-ESPRIT algorithm applying the subspace approach which decreases the errors caused by the model nonlinearity effect and secondly due to Doppler correction, which reduces the DOAE uncertainty associated with the effect of the target movement.

The integrated analysis for Figures 6 and 9 showed us that the proposed algorithm not only enhanced the accuracy of the estimation process, but also illuminated the effect of 


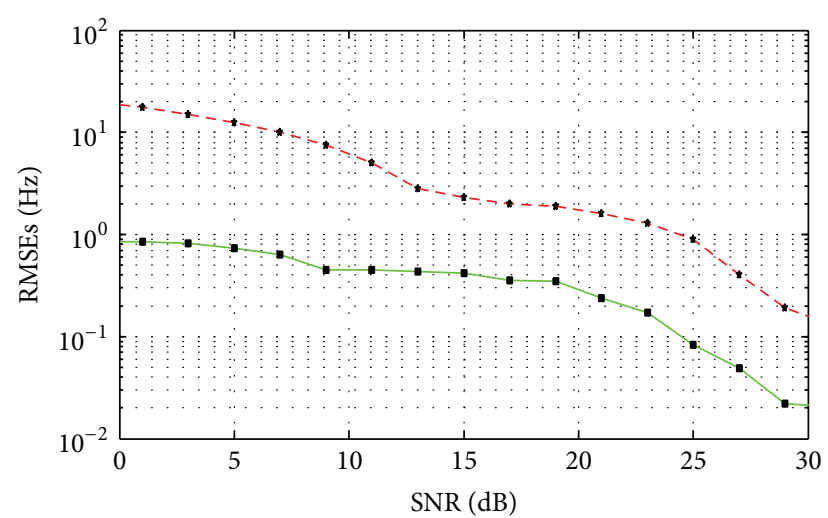

- T-DIT

- - Reference [20]

FIGURE 7: Estimated Doppler frequency RMSEs of T-DIT method.

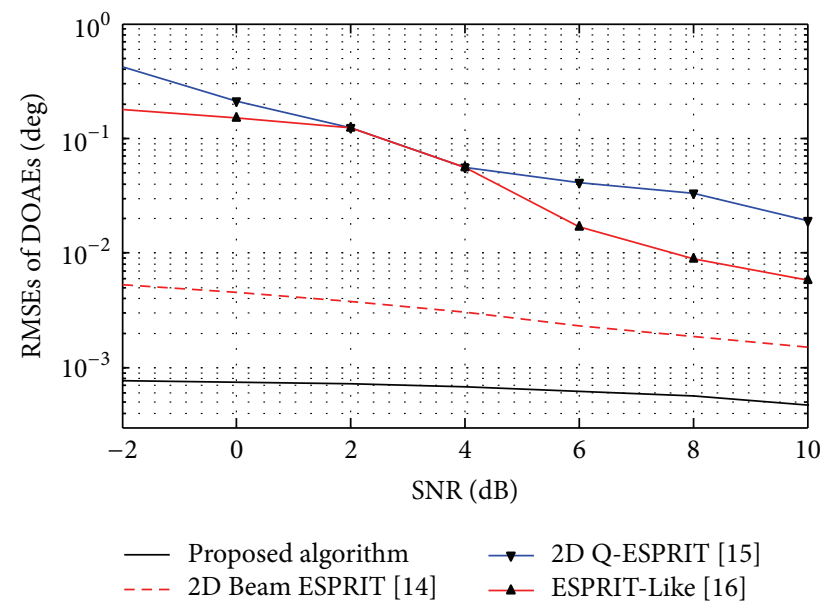

FIgURE 8: RMSEs versus SNR for T-ESPRIT with the Doppler effect embedding compared with different ESPRIT methods.

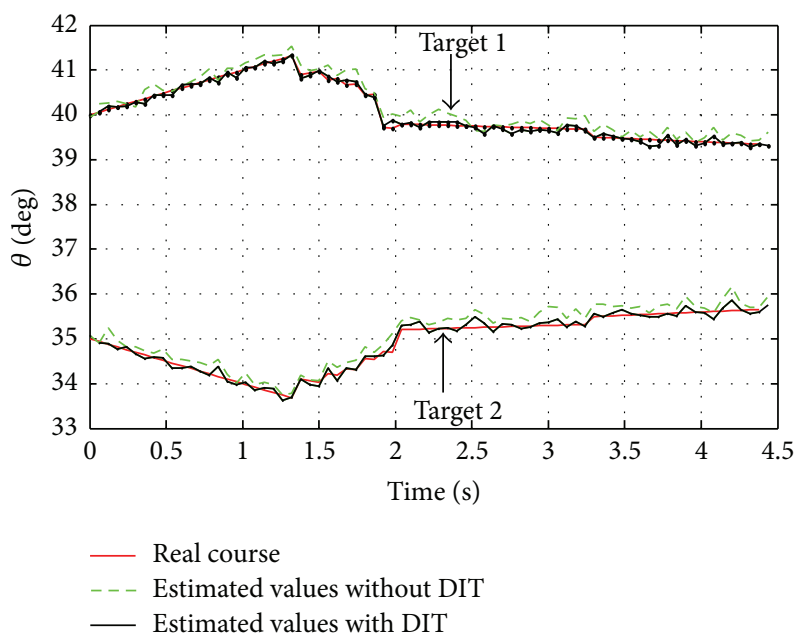

FIGURE 9: The moving target elevation angles during flight course.

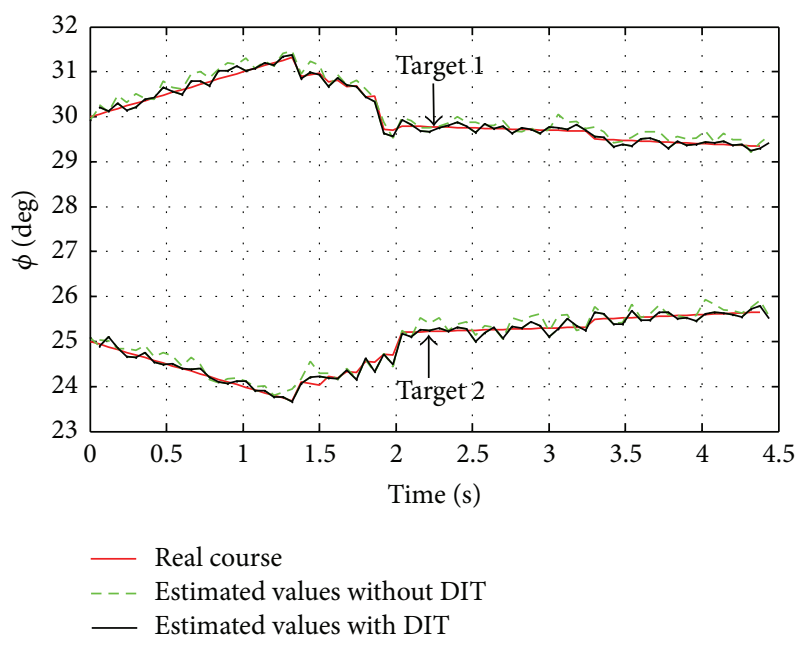

FIGURE 10: The moving target azimuth angles during flight course.

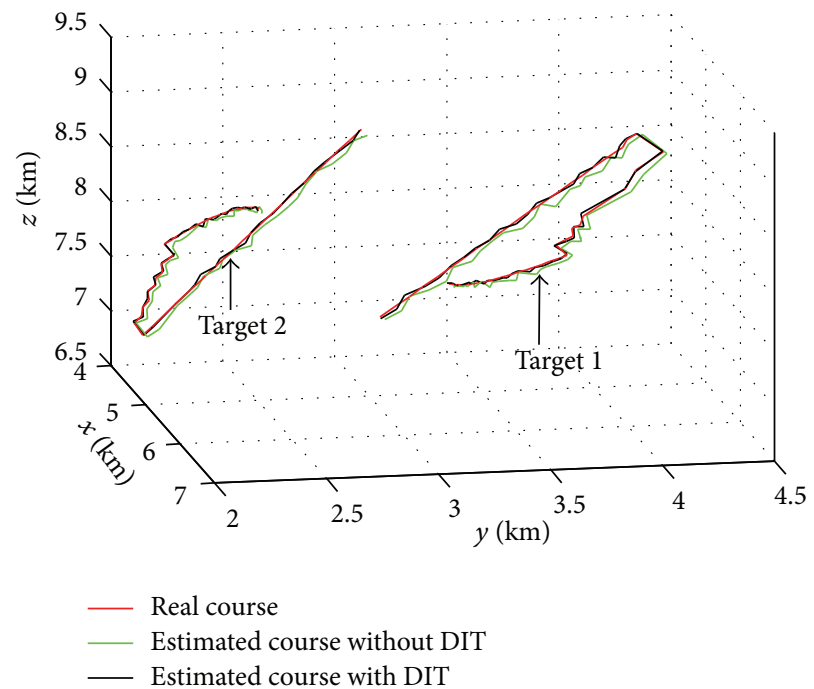

FIGURE 11: The moving target flight course.

target maneuver on the estimation accuracy, which revealed the Doppler frequency effect on the estimation process.

Thus, the 2D-DOAE accuracy has been improved by embedding the Doppler effect that resulted from target movement into T-ESPRIT algorithm. For more validation, this algorithm has been applied for two targets moving with different courses to highlight the good performance of this method; the first target is moving away from the antenna then turns toward it with initial value of $\alpha=85^{\circ}$. On the contrary, the second target moving course started with moving toward the antenna then moving away from it with initial value of $\alpha=265^{\circ}$ and $\dot{\alpha}=3^{\circ} / \mathrm{sec}$ for both of them. Comparison between true and estimated angles $(\theta, \varphi)$ of the moving two targets is shown at Figures 9 and 10, respectively. Figure 11 showed comparison between true and estimated flight course plots of the two moving targets. Clearly, we can figure out that the process of integration between DIT algorithm to estimate Doppler frequency and the modified 
ESPRIT algorithm makes the estimated flight course more close to the real one, which means less angle glint error (almost up to $0.15^{\circ}-0.3^{\circ}$ ) as shown in Figures 9 and 10 . Simply, we can say that the developed algorithm achieved success in increasing the DOAE accuracy with low computational load leading to the increase of the estimator efficacy.

From Table 3, it has been found that the proposed algorithm requires $O\left(2\left[M+4 r(I L)^{2}+8(I L)^{3}\right]\right)$ flops, while the conventional ESPRIT algorithm needs $O\left(2\left[4 d(I L)^{2}+\right.\right.$ $\left.\left.8(I L)^{3}\right]\right)$ flops. The developed ESPRIT algorithm requires about $57.6 \%$ of the computational time compared with that of the classical ESPRIT algorithm. Simply, it has been found that the developed ESPRIT method achieved success in increasing the DOAE accuracy with low computational load leading to the increase of the estimator efficacy.

\section{Conclusions}

The DOA estimator performance is affected by target maneuver and nonlinear relationships between the measured quantities and the desired target coordinates.

In order to reduce the DOAE errors, the DOA estimated values should be corrected according to the target maneuver. From our point of view, Doppler effect is the best way that can be used to describe the effect of target maneuver on DOAE process because the correction can be applied according to the fluctuation of the echo signal wavelength change from the transmitted one induced by the target maneuver. Lack of attention to the influence of Doppler shift on the phase shift measurement in DOAE process causes angle glint error and consequently leads to DOAE process with poor accuracy.

In this paper, a new algorithm for 2D-DOAE, which aspires to contribute to the increase of the estimation process accuracy and the decrease of the computational load, has been firstly carried out. It has introduced time multiresolution in ESPRIT method to realize temporal subspace approach (T-ESPRIT) that decreases errors caused by the model's nonlinearity effect and to reduce the computational load via processing the temporal subspaces in parallel, which leads to the shrinkage of the covariance matrix dimension. Additionally, the subspace approach has been employed into DIT method in order to form T-DIT technique to reduce the Doppler frequency estimation computational costs. Secondly, in order to curb target maneuver effect on the DOAE process, an equation that describes the effect of Doppler frequency on the ESPRIT method has been derived to realize the DOAE correction.

The proposed algorithm has less computational complexity; it requires $O\left(2\left[M+4 r(I L)^{2}+8(I L)^{3}\right]\right)$ flops. Also, it reduces $88.91 \%$ of the computational time of Doppler estimation via PWZFFT method and $42.37 \%$ of the $2 \mathrm{D}$-DOAE total computational time with Doppler correction implementation. Additionally, the proposed algorithm realizes less angle glint error (almost up to $0.15^{\circ}-0.3^{\circ}$ ). Finally, it has been found that the estimation accuracy has been increased with low computational load, and it consequently enhances the estimator performance.

\section{Conflict of Interests}

The authors declare that there is no conflict of interests regarding the publication of this paper.

\section{Acknowledgments}

The authors would like to acknowledge that this work was supported in part by the National Natural Science Foundation of China (no. 61301211) and the Key Laboratory of Radar Imaging and Microwave Photonics (Nanjing University of Aeronautics and Astronautics), Ministry of Education, Nanjing University of Aeronautics and Astronautics, Nanjing, China.

\section{References}

[1] R. Roy, A. Paulraj, and T. Kailath, "ESPRIT-estimation of signal parameters via rotational invariance techniques," in Proceedings of the IEEE International Conference on Acoustics, Speech, and Signal Processing (ICASSP '86), vol. 11, pp. 2495-2498, Tokyo, Japan, 1986.

[2] R. H. Roy, ESPRIT-estimation of signal parameters via rotational invariance techniques [Ph.D. thesis], Stanford University, Stanford, Calif, USA, 1987.

[3] R. Roy and T. Kailath, "ESPRIT-estimation of signal parameters via rotational invariance techniques," IEEE Transactions on Acoustics, Speech, and Signal Processing, vol. 37, no. 7, pp. 984995, 1989.

[4] Z. I. Khan, R. A. Awang, A. A. Sulaiman et al., "Performance analysis for estimation of signal Parameters via Rotational Invariance Technique (ESPRIT) in estimating direction of arrival for linear array antenna," in Proceedings of the IEEE International RF and Microwave Conference (RFM '08), pp. 530-533, IEEE, Kuala Lumpur, Malaysia, December 2008.

[5] Y. Fayad, C. Wang, A. E.-D. S. Hafez, and Q. Cao, "Direction of arrival estimation using novel ESPRIT method for localization and tracking radar systems," in Proceedings of the 11th International Bhurban Conference on Applied Sciences and Technology (IBCAST '14), pp. 396-398, IEEE, Islamabad, Pakistan, January 2014.

[6] Y. Fayad, C. Wang, Q. Cao, and A. E.-D. S. Hafez, "A developed ESPRIT algorithm for DOA estimation," Frequenz, vol. 69, no. 3, pp. 263-269, 2015.

[7] V. Vasylyshyn, "Direction of arrival estimation using ESPRIT with sparse arrays," in Proceedings of the EUMA-6th European Radar Conference, Rome, Italy, September 2009.

[8] F. Gao and A. B. Gershman, "A generalized ESPRIT approach to direction-of-arrival estimation," IEEE Signal Processing Letters, vol. 12, no. 3, pp. 254-257, 2005.

[9] G.-M. Xu and J.-G. Huang, "Multi-resolution parameters estimation for polarization sensitivity array," in Proceedings of the IEEE International Symposium on Knowledge Acquisition and Modeling Workshop (KAM '08), pp. 180-183, December 2008.

[10] A. N. Lemma, A.-J. van der Veen, and E. F. Deprettere, "Joint angle-frequency estimation using multi-resolution ESPRIT," in Proceedings of the IEEE International Conference on Acoustics, Speech and Signal Processing (ICASSP '98), vol. 4, pp. 1957-1960, May 1998. 
[11] A. N. Lemma, A.-J. D. van der Veen, and E. F. Deprettere, "Multiresolution ESPRIT algorithm," IEEE Transactions on Signal Processing, vol. 47, no. 6, pp. 1722-1726, 1999.

[12] A. L. Swindlehurst, B. Ottersten, R. Roy, and T. Kailath, "Multiple invariance ESPRIT," IEEE Transactions on Signal Processing, vol. 40, no. 4, pp. 867-881, 1992.

[13] Y. Zhang, Z. Ye, X. Xu, and J. Cui, "Estimation of twodimensional direction-of-arrival for uncorrelated and coherent signals with low complexity," IET Radar, Sonar and Navigation, vol. 4, no. 4, pp. 507-519, 2010.

[14] C. P. Mathews, M. Haardt, and M. D. Zoltowski, "Performance analysis of closed-form, ESPRIT based 2-D angle estimator for rectangular arrays," IEEE Signal Processing Letters, vol. 3, no. 4, pp. 124-126, 1996.

[15] Y. Li, J. Q. Zhang, B. Hu, H. Zhou, and X. Y. Zeng, "A novel 2-D quaternion ESPRIT for joint DOA and polarization estimation with crossed-dipole arrays," in Proceedings of the IEEE International Conference on Industrial Technology (ICIT '13), pp. 1038-1043, Cape Town, South Africa, February 2013.

[16] F.-J. Chen, S. Kwong, and C.-W. Kok, "ESPRIT-like twodimensional DOA estimation for coherent signals," IEEE Transactions on Aerospace and Electronic Systems, vol. 46, no. 3, pp. 1477-1484, 2010.

[17] H. Jiang and G. Yang, "An improved algorithm of ESPRIT for signal DOA estimation," in Proceedings of the International Conference on Industrial Control and Electronics Engineering (ICICEE '12), pp. 317-320, August 2012.

[18] X. Xu and Z. Ye, "Two-dimensional direction of arrival estimation by exploiting the symmetric configuration of uniform rectangular array," IET Radar, Sonar and Navigation, vol. 6, no. 5, pp. 307-313, 2012.

[19] V. C. Chen, The Micro-Doppler Effect in Radar, Artech House, Norwood, Mass, USA, 2011.

[20] C. Yunhe, "Joint estimation of angle and Doppler frequency for bistatic MIMO radar," Electronics Letters, vol. 46, no. 2, pp. 170 $172,2010$.

[21] F. B. Berger, “The nature of Doppler velocity measurement," IRE Transactions on Aeronautical and Navigational Electronics, vol. 4, no. 3, pp. 103-112, 1957.

[22] A. Nabavizadeh, M. W. Urban, R. R. Kinnick, and M. Fatemi, "Velocity measurement by vibro-acoustic Doppler," IEEE Transactions on Ultrasonics, Ferroelectrics, and Frequency Control, vol. 59, no. 4, pp. 752-765, 2012.

[23] A-level Physics (Advancing Physics), http://en.wikibooks.org.

[24] A. N. D’Andrea, U. Mengali, and R. Reggiannini, “The modified Cramer-Rao bound and its application to synchronization problems," IEEE Transactions on Communications, vol. 42, no. 2, pp. 1391-1399, 1994.

[25] S. T. Smith, "Statistical resolution limits and the complexified Cramér-Rao bound," IEEE Transactions on Signal Processing, vol. 53, no. 5, pp. 1597-1609, 2005.

[26] R. Tao, W.-Q. Zhang, and E.-Q. Chen, “Two-stage method for joint time delay and Doppler shift estimation," IET Radar, Sonar and Navigation, vol. 2, no. 1, pp. 71-77, 2008. 

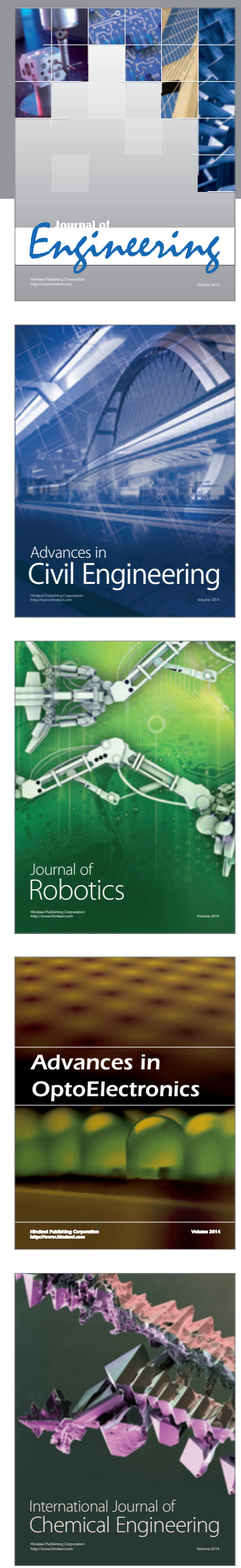

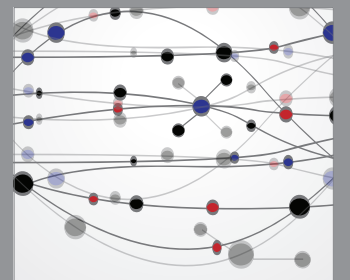

The Scientific World Journal
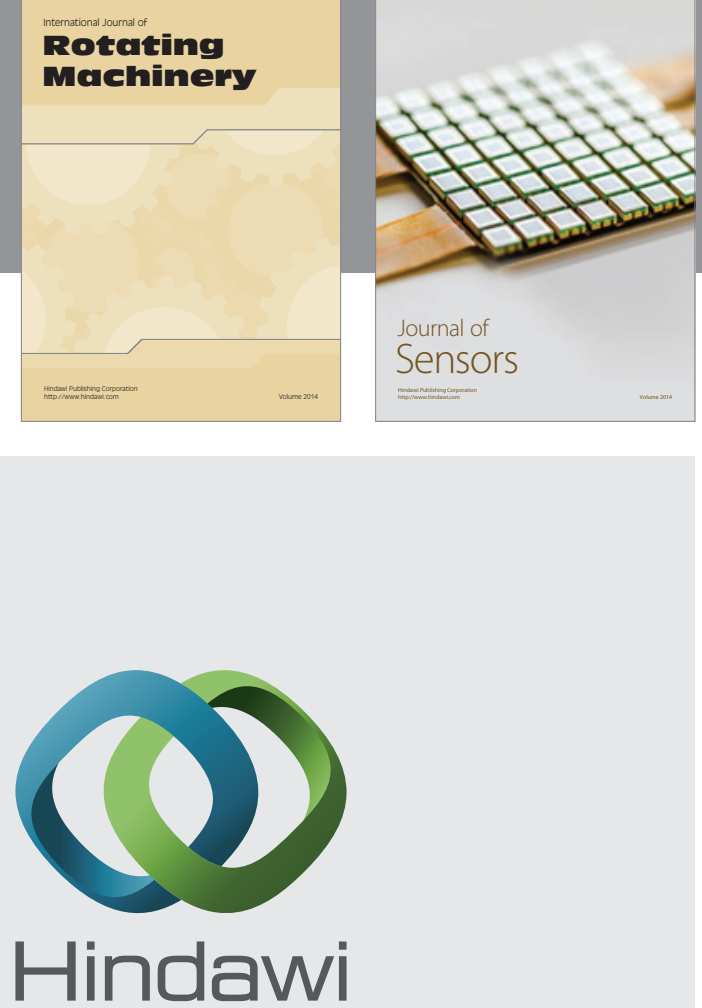

Submit your manuscripts at http://www.hindawi.com
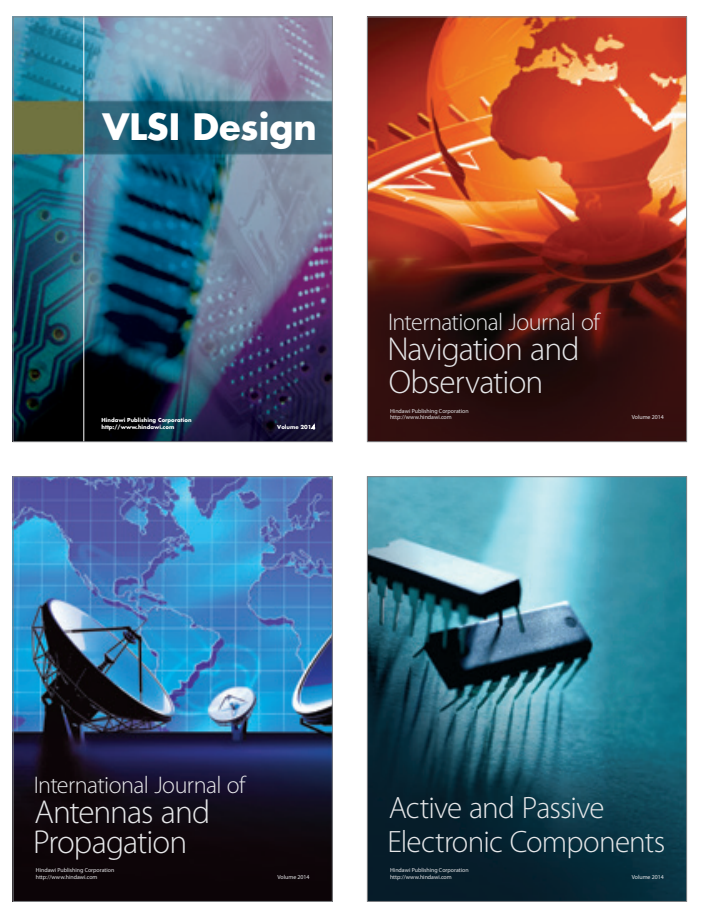
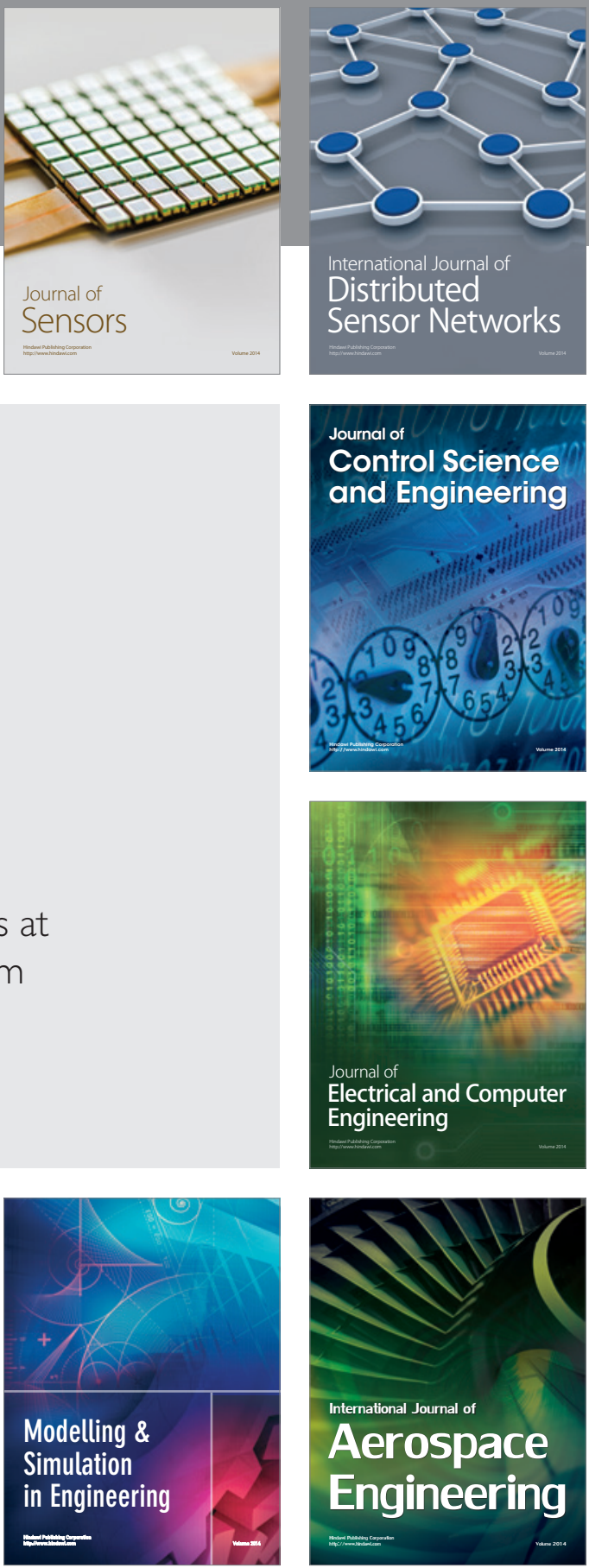

Journal of

Control Science

and Engineering
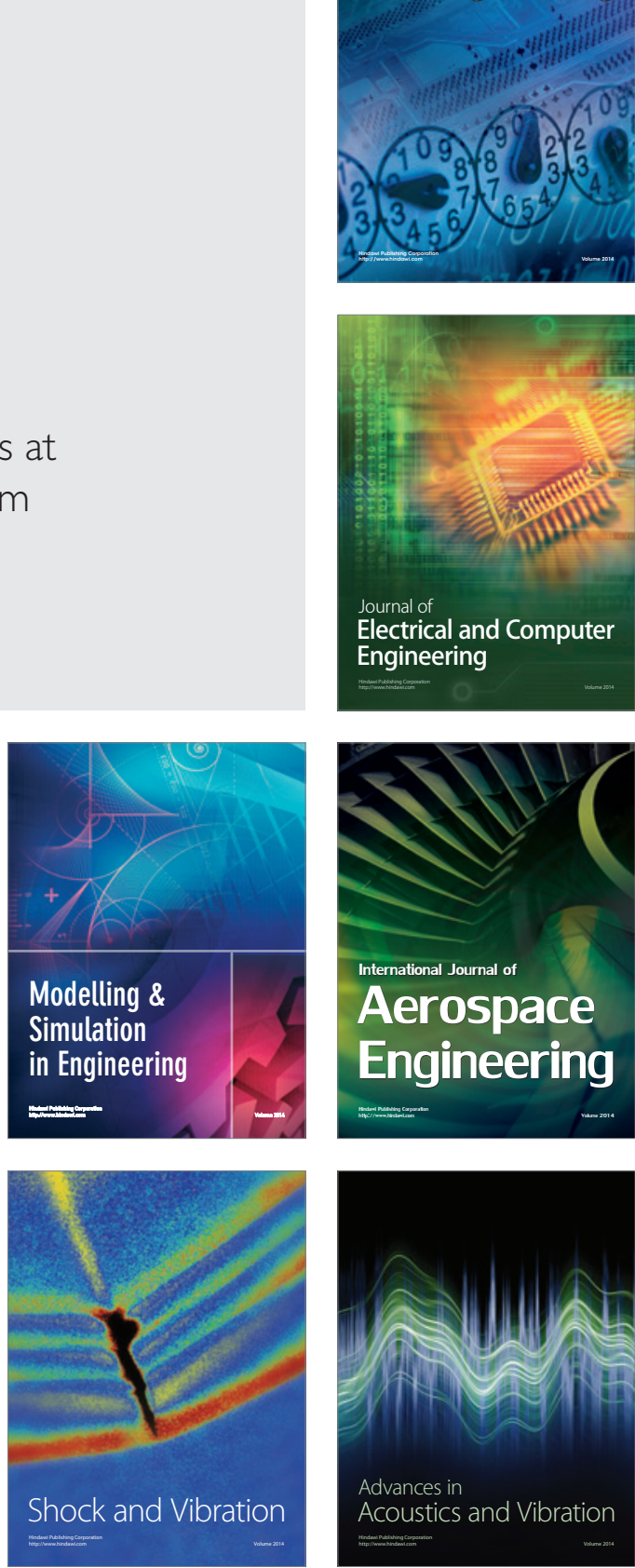